\title{
Telephone-guided self-help for mental health difficulties in neurological conditions: a randomised pilot trial
}

Sophie D. Bennett, Senior Research Fellow, UCL Great Ormond Street Institute of Child Health \& Honorary Clinical Psychologist, Great Ormond Street Hospital for Children NHS Foundation Trust

Isobel Heyman, Consultant Child and Adolescent Psychiatrist, Great Ormond Street Hospital for Children NHS Foundation Trust \& Honorary Professor, UCL Great Ormond Street Institute of Child Health

Anna E. Coughtrey, Clinical Research Fellow and Clinical Psychologist, Great Ormond Street Hospital for Children NHS Foundation Trust and UCL Great Ormond Street Institute of Child Health

Sophia Varadkar, Consultant Paediatric Neurologist, Great Ormond Street Hospital for Children NHS Foundation Trust \& Honorary Senior Lecturer, UCL Great Ormond Street Institute of Child Health

Terence Stephenson, Nuffield Professor of Child Health, UCL Great Ormond Street Institute of Child Health

Epilepsy and Mental Health Programme Development Grant Research Group ${ }^{+}$

Roz Shafran, Professor of Translational Psychology, UCL Great Ormond Street Institute of Child Health \& Honorary Consultant Clinical Psychologist, Great Ormond Street Hospital for Children NHS Foundation Trust

*Corresponding author: UCL Great Ormond Street Institute of Child Health, 30 Guilford Street, London, WC1N 1EH; Sophie.bennett.10@ucl.ac.uk; ORCID: https://orcid.org/00000002-1076-7112

+Marta Buszewicz, Sarah Byford, Caroline Dore, Peter Fonagy, Tamsin Ford, Rona MossMorris, Susan Tebbs \& Erin Walker

Word count: 2499

Trial registration: Current Controlled Trials register (ISRCTN21184717). 


\section{Abstract}

Objective: This study aimed to conduct a randomised pilot trial to assess the feasibility of an RCT to investigate the effect of telephone-guided self-help for the treatment mental health difficulties in children with neurological conditions.

Design: Preliminary Randomised Controlled Trial. The primary outcome measure was the Strengths and Difficulties Questionnaire.

Setting: Neurology clinics in a national tertiary paediatric hospital.

Patients: Young people attending neurology clinics who met criteria for mental health difficulties according to the Development and Wellbeing Assessment (DAWBA).

Interventions: 12 weeks of telephone-delivered telephone-guided self-help based on a modular approach to psychological therapy for children $(n=17 ; 8$ males; mean age 12.04 years, SD 3.34) or a waiting list for telephone-guided self-help with no additional intervention over 12 weeks ( $\mathrm{n}=17 ; 9$ males; mean age 10.53 years, SD 3.14).

Results: $47 \%$ of patients screened met diagnostic criteria for a mental health disorder. $65 \%$ of those randomised to the intervention arm completed the full intervention and the intervention was acceptable to those completing it. However, there were significant problems related to lack of data completion (38\% data loss for primary outcome measure), choice of control comparator and outcome measures. Due to significant loss of data to follow-up, the effect size findings are considered unreliable.

Conclusions: Further feasibility work should be conducted to improve data completeness before progression to a definitive trial of guided self-help for mental health problems in children with neurological conditions can be recommended. 
Despite the high prevalence of mental health disorders in children and young people with neurological conditions and associated adverse outcomes, they are frequently not detected or effectively treated ${ }^{1}$. For example, in epilepsy (the largest patient group covered by paediatric neurolog$y^{2}$ ) the proportion of children with mental health problems who are in receipt of any mental health care has been estimated at between only $20 \%$ and $50 \%{ }^{3,4}$ and parents of children with epilepsy are more likely to report unmet need for mental health services than those of children without epilepsy ${ }^{5}$. As such, there are increasing calls for routine screening for mental health problems in children with epilepsy ${ }^{6}$. Researchers have also recommended routine mental health assessment and treatment for children with Cerebral Palsy and neurovascular conditions due to the high prevalence of mental health disorders in these groups $^{7-9}$ and nationally, there is an increasing drive to integrate physical and mental healthcare across all chronic illnesses ${ }^{10}$.

Guided self-help interventions can be convenient methods of delivering therapy for families and for services and are now a recommended first-line treatment for a number of mental health difficulties in adults and children ${ }^{11,12}$. They are low cost, more accessible and similar in efficacy to face-to-face treatment in children and young people with mental health disorders ${ }^{13}$. Such remotely delivered approaches may be particularly beneficial in the context of the current pandemic. Whilst guided self-help and standard face-to-face therapy have demonstrated equivalence in children and young people, this has not been shown specifically for children with mental health needs in the context of neurological conditions; indeed, there has been very little investigating mental health intervention for children with neurological conditions using any evidence-based treatment ${ }^{14,15}$. It is important to ensure that future trials investigating the efficacy of guided self-help for children with neurological conditions are feasible with regards to both study processes and intervention. In particular, it is important to determine the feasibility of recruitment and data collection in paediatric services given 
families have numerous existing demands on their time, and the feasibility of intervention given the lack of literature on the effectiveness and acceptability of mental health interventions for this group. This study therefore aimed to conduct a randomised pilot trial to assess the feasibility of an RCT to investigate the effect of telephone-guided self-help for the treatment mental health difficulties in children with neurological conditions. In line with guidance on feasibility and pilot trials, ${ }^{16}$ the objectives were to investigate the following:

1. Whether the components of the study worked effectively together, including recruitment procedures, data collection procedures and intervention procedures

2. Recruitment and retention rates: can patients be recruited and what proportion go on to complete the intervention and measures?

3. The acceptability to young people and families of the study design and the intervention

4. The preliminary expected effect size for the primary outcome measure

\section{Method}

The published protocol for this pilot study is available ${ }^{17}$. The study was registered with the Current Controlled Trials register (ISRCTN21184717).

\section{Intervention}

\section{Telephone-delivered Guided self-help (GSH) arm}

The telephone-delivered guided self-help was based on the Modular Approach to Therapy for Children with Anxiety, Depression, Trauma, or Conduct Problems (MATCH$\mathrm{ADTC}^{18}$ ). Adaptations were made for delivery in a self-help format, in which worksheets were sent to participants prior the session and support was provided through 10 weekly 30 minute telephone calls delivered within 12 weeks. The intervention was delivered to the 
parents and/or young person depending on presenting difficulty, age and intellectual ability.

\section{Waiting-List Control (WLC) arm}

Children were on the waiting list for 12 weeks and did not receive any psychological intervention during this time. At the end of the 12 weeks, they completed the SDQ and then received the telephone-guided self-help intervention for 12 weeks.

\section{Measures}

All outcome measures were parent-reported. The primary outcome measure was the Strengths and Difficulties Questionnaire (SDQ) total $^{19,20}$ - a 25-item psychometrically robust questionnaire used for the identification of common mental and behavioural symptoms in young people. After the intervention, parents also completed the Experience of Service Questionnaire (ES $\left.Q^{21}\right)$ - comprising 12 items investigating experience of service (e.g. 'I was treated well by the people who have seen my child') rated on a three-point scale (not true, partly true, certainly true). Additional measures are reported in a supplementary document, which details all secondary outcomes and results and discussion of these measures.

\section{Procedure}

The study implemented a routine voluntary screening programme within paediatric neurology clinics at a national paediatric hospital. Information leaflets regarding the study were mailed to families of children aged 3-18 together with their clinic appointment information. Families who were in the clinic waiting room were then approached by a research assistant regarding the study. Those providing informed consent were then asked to complete the parent-report SDQ on a tablet. This was scored automatically. Those reaching a pre-specified threshold (a combination of raised symptom score $-\geq 14$ out of a maximum of 40 , which is a score in the 'slightly raised' range or higher; and raised impact score $-\geq 2$ out 
of a maximum of 10 indicating a score in the 'high' range or above ${ }^{22}$ ) were invited to proceed to completing the full Development and Wellbeing Assessment (DAWBA) ${ }^{23}$ at home and given instructions for accessing this. The DAWBA is a package of interviews, questionnaires and rating techniques designed to generate ICD-10 and DSM-IV or DSM-5 psychiatric diagnoses on 5-17 year olds and available for parents to complete independently online. These procedures are detailed further in a paper reporting on the identification of mental health difficulties in paediatric neurology $\operatorname{clinics}^{24}$.

Children were excluded if (1) they had an intellectual disability at a level meaning that parents felt that the screening measures did not apply to them, (2) there were high levels of risk (for example suicidal intent), (3) they had a mental health disorder other than depression, anxiety, or disruptive behaviour which required immediate treatment (e.g. psychosis), (4), they were undertaking frequent on-going psychological treatment for an emotional or behavioural problem, or (5) the parent/carer was unable to speak/understand English sufficiently well to access the screening assessments and interventions.

Participants experiencing impairing symptoms of mental health disorder according to the DAWBA were randomised to: (a) telephone-delivered guided self-help for their emotional or behavioural difficulties over 12 weeks (GSH group) or (b) remaining on the waiting list for telephone-delivered guided self-help with no additional intervention over 12 weeks (waiting list control group). Stratified by primary mental health disorder (anxiety/depression/disruptive behaviour disorder), participants were randomised 1:1 to treatment arm using random blocks of three/four created by www.sealedenvelope.com. An independent researcher who was not otherwise involved in the assessment procedure held and provided the randomisation codes. 
The associated identification study was funded for 12 months and we aimed to recruit at least 18 participants based on estimated screening rates and retention rates in this time $\operatorname{period}^{25}$.

\section{Data analysis}

Data associated with the four study objectives were analysed as follows:

\section{Whether the components of the study worked effectively together}

Time taken to complete primary outcome measure, average length of support sessions, adherence to the intervention protocol and study protocol deviations were recorded. Therapist adherence to the intervention protocol was assessed by trained independent raters using a bespoke adherence measure. Treatment completion was calculated as the percentage of participants in the GSH group completing 9 or 10 sessions.

\section{Recruitment and retention rates}

We obtained descriptive estimates of the number of eligible participants, number consenting, numbers completing the intervention and numbers completing the outcome measures at each time point.

3. The acceptability to young people and families of the study design and the intervention

The mean score for the ESQ Satisfaction with Care subscale was calculated.

4. The expected effect size for the primary outcome measure

Treatment groups were descriptively compared on all baseline measures. Difference scores were based on the mean change in scores between baseline and 12-week post-baseline assessment scores on the SDQ; these were converted into standardised effect sizes (Cohen's d). It was originally planned that these would be based on Intent to Treat data using Multiple Imputation, but only completer data was analysed due to the high proportion of missing data. 


\section{Ethics}

The study received ethical approval from the Camden and Islington Research Ethics Committee (registration number 14.LO.1353). Written informed consent for participation was obtained from participating young people and their families.

\section{Results}

1. Whether the components of the study worked effectively together

There were no significant adverse events. The SDQ took less than 10 minutes for participants to complete. Regarding protocol deviations, many participants completed either the 12 or the 16-week post-baseline measures. Therefore, these have been amalgamated into one 'post' score (T2; presented in figure 1). The 12-week post-baseline measure was used where available, with the 16-week post-baseline score replacing this value if it was missing. This "T2" measure was completed an average of 110 days (15.75 weeks) following baseline (T1). T3 refers to those completed 24 weeks postbaseline.

The average length of sessions 1-10 of the 175 recorded treatment sessions was 34 minutes ( $\mathrm{SD}=5$ minutes). All participants were considered to have received sessions that adhered to the protocol and no major 'off-protocol' content was noted.

\section{Recruitment and retention rates}

Figure 1 outlines the flow of participation through the study, based on completion of the primary outcome measure (parent-completed SDQ). 675 families were approached in clinic, of whom 419 consented to take part in the screening study. 406 of these completed the SDQ. 232 of those completing the SDQ met the threshold for continuation and were invited to complete the DAWBA. 124 participants completed the DAWBA and 34 people were entered into the trial. Seventeen participants were randomised into each arm. Participant 
characteristics are outlined in Table 1. A greater proportion of children in the WLC arm had symptoms of, or met diagnostic criteria, for Autism Spectrum Disorder compared to those in the GSH group (65\% vs. 35\%, respectively). The number of psychiatric diagnoses per participant ranged from 0-3. The median number in the telephone-guided self-help arm was 1 (range 1-3; IQR = 1) and in the Waiting List Control arm was 2 (range 0-3; IQR = 1).

23 participants $(68 \%)$ completed the intervention (11 in the intervention arm $(65 \%)$ and 12 in the WLC arm who subsequently were offered the intervention (71\%)). Three of the participants who discontinued the intervention were later contacted through an associated qualitative study and reported that they prematurely ended the intervention due to not finding it helpful $(n=1)$, lack of rapport with the therapist $(n=1)$ and finding the intervention to be too much work $(\mathrm{n}=1)^{26}$.

Seven out of the 17 participants in the GSH arm and 14 of the 17 participants in the WLC arm completed the SDQ at T2. 7 of the 17 participants in the GSH group completed the SDQ at T3.

\section{The acceptability to young people and families of the study design and the intervention}

The mean ESQ score for the satisfaction with care subscale was 16.875 (SD $=1.26$; range 14-18) out of 18 in the 16 participants who completed it following the intervention, indicating a high level of satisfaction ${ }^{21}$.

\section{Expected effect size of the primary outcome measure}

The means and standard deviations for those randomised in the trial who completed the SDQ at both time-points are summarised in Table 2. The mean SDQ Total scores for the GSH fell 2.72 points from $21.43(\mathrm{SD}=3.26)$ at $\mathrm{T} 1$ to $18.71(\mathrm{SD}=2.14)$ at $\mathrm{T} 2$, demonstrating improvement in symptoms. Those in the WLC fell 2.78 points from 22.07 (3.69) to 19.29 $(\mathrm{SD}=4.70)$, also demonstrating improvement. This represents a between groups difference in 
T1-T2 change of 0.06 SDQ points in favour of the WLC. The between groups difference at T2 was 0.58 , in favour of the GSH arm (Cohen's d effect size of $0.16,95 \%$ CI [-0.90;1.20]).

\section{Discussion}

This study aimed to conduct a randomised pilot trial to assess the feasibility of an RCT to investigate the effect of telephone-guided self-help for the treatment mental health difficulties in children with neurological conditions. Overall, the results suggest that further research examining different outcomes, recruitment pathways and control groups is needed before proceeding to a full-scale trial of this guided self-help intervention.

There was significant interest in the study from parents and young people, reflected in the high uptake of the initial screening. It appears that this high uptake is needed to ensure throughput into the final study, as a number of participants were lost to follow-up following each stage of the screening process. The availability of time, space and/or equipment for families to complete the online DAWBA may be a limitation of this approach. The vast majority of patients in the trial were those with epilepsy, reflecting the greater numbers of patients in neurology clinics with epilepsy compared to other neurology clinics. A more targeted process, for example through referrals directly from the physical healthcare team, may result in a higher percentage of those consenting to the overall study going on to complete the intervention.

The families completing the measures and intervention found these to be acceptable according to the Experience of Service Questionnaire but little data were collected from those who did not complete the intervention and/or measures. $65 \%$ of GSH participants completed the full intervention but almost $40 \%$ did not complete the primary outcome. The high proportion of missing data and amalgamation of the 12-week follow-up and 16-week followup data requires consideration prior to further trials and means that effect size data are 
considered unreliable. Data loss of a maximum of $20 \%$ would be needed to proceed to a definitive trial $^{27}$. The high proportion of missing data may be due to this group of parents having multiple stressors and the remote method of intervention delivery and data collection. Focus groups with families may help to identify measures and methods of data collection that capture the necessary information without over-burdening families. It may be that an intervention with more therapist input, fewer measures and simpler methods of remote data collection would have lower attrition ${ }^{28}$. Future studies may also benefit from teacher-rated measures, or measures blinded to the participant's status. These findings have informed subsequent related studies, with greater measure completion rates ${ }^{28,29,30}$.

The within group effect size for the SDQ total was large but the overall between groups effect size was small (below 0.2) as symptoms also improved during the WLC period. One reason for the decrease may be that completion of the in-depth diagnostic interview (DAWBA) was therapeutic, particularly as this was often the first time that any of the patients had received any assessment for their mental health. It is possible that changes reflect regression to the mean, particularly given the slightly higher baseline SDQ scores for the WLC arm. The improvement in SDQ total in the WLC may also be an anomaly due to the small sample size and very high proportion of missing data, which means the effect sizes calculated are unreliable.

The learning from this study should be used to inform future research of this telephone-delivered self-help intervention for children and young people with neurological conditions.

\section{Acknowledgements}

The authors would like to thank Professor Robert Goodman for the use of the SDQ and DAWBA. We also thank the neurologists and staff in paediatric neurology clinics at 
Great Ormond Street Hospital for Children NHS Foundation Trust for their support with the study. We would also like to acknowledge the Mental Health in Children with Epilepsy (MICE) study team. This work was funded by the Great Ormond Street Hospital Children's Charity (Grant Number 17663). The research was supported by the National Institute for Health Research Biomedical Research Centre at Great Ormond Street Hospital for Children NHS Foundation Trust and University College London. The views expressed are those of the authors and not necessarily those of the NHS, the NIHR or the Department of Health.

\section{Contributors}

SB was a study co-investigator, the project management lead, was the therapist for majority of patients and wrote the first draft of the manuscript for publication. IH was the joint lead co-applicant and Great Ormond Street Hospital psychiatric and clinical lead, designed the study and supervised DAWBA ratings. AC was a study co-investigator, contributed to the overall design of the study and rated all DAWBAs. SV was a study coinvestigator, was the neurology lead and supported study management in the neurology clinics. TC was a study co-investigator, paediatric consultant and contributed to study design. RS was the joint lead co-applicant, designed the study, was therapist for some patients and supervisor of therapy for all and supervised the management of the study. SB and RS had complete access to the study data that support the publication.

\section{What is already known on this topic}

- Despite the high prevalence of mental health disorders in children with neurological conditions and associated adverse outcomes, they are frequently not detected or effectively treated. 
- Guided self-help is a convenient way to increase access to therapy and recommended first-line treatment for several mental health difficulties in adults and children.

- Guided self-help and telephone-delivered interventions may have particular value within a paediatric setting in order to avoid repeated travel to the clinic for face-toface appointments.

\section{What this study adds}

- The learning from this study should be used to inform future research of this telephone-delivered self-help intervention for children and young people with neurological conditions.

- The findings indicate issues related to study design, including choice of outcome measure, methods of data collection, intervention and control group.

- Further research considering the therapeutic value of assessment, recruitment strategies and appropriate outcome measures for remotely delivered studies in children and young people is needed. 


\section{References}

1. Pattanayak RD, Sagar R. Psychiatric aspects of childhood epilepsy. Iranian Journal of Child Neurology. 2012;6:9-18.

2. NHS England NHS England, 2018 https://www.england.nhs.uk/wpcontent/uploads/2018/09/E09-S-b-Paediatric-Neurosciences-Neurology.pro_.2013.04.v2.pdf)

3. Hanssen-Bauer K, Heyerdahl S, Eriksson A-S. Mental health problems in children and adolescents referred to a national epilepsy center. Epilepsy \& Behavior. 2007;10:255-62.

4. Ott D, Siddarth P, Gurbani S, et al. Behavioral disorders in pediatric epilepsy: unmet psychiatric need. Epilepsia. 2003;44:591-7.

5. Russ SA, Larson K, Halfon N. A national profile of childhood epilepsy and seizure disorder. Pediatrics. 2012;129:256-64.

6. Reilly C, Atkinson P, Das KB, et al. Neurobehavioral comorbidities in children with active epilepsy: a population-based study. Pediatrics. 2014;133:e1586-93.

7. Bjorgaas H, Elgen I, Boe T, Hysing M. Mental health in children with cerebral palsy: does screening capture the complexity? The Scientific World Journal. 2013;2013.

8. Downs J, Blackmore AM, Epstein A, et al. The prevalence of mental health disorders and symptoms in children and adolescents with cerebral palsy: a systematic review and metaanalysis. Developmental Medicine \& Child Neurology. 2018;60:30-8.

9. O'Keeffe F, Stark D, Murphy O, Ganesan V, King J, Murphy T. Psychosocial outcome and quality of life following childhood stroke-A systematic review. Developmental neurorehabilitation. 2017;20:428-42.

10. Naylor C, Das P, Ross S, Honeyman M, Thompson J, Gilburt H. Bringing together physical and mental health. King's Fund. 2016. 
11. National Institute for Health and Care Excellence. Depression in children and young people: identification and management (NICE Clinical Guidelines No. 28). 2017 [ONLINE] https://www.nice.org.uk/guidance/cg28

12. National Institute for Health and Care Excellence. Attention deficit hyperactivity disorder (NICE Quality Standard No. 39). 2013 [ONLINE] https://www.nice.org.uk/guidance/qs39

13. Bennett SD, Cuijpers P, Ebert DD, et al. Practitioner Review: Unguided and guided self-help interventions for common mental health disorders in children and adolescents: a systematic review and meta-analysis. Journal of Child Psychology and Psychiatry. 2019;60:828-47.

14. Bennett S, Shafran R, Coughtrey A, Walker S, Heyman I. Psychological interventions for mental health disorders in children with chronic physical illness: a systematic review. Archives of disease in childhood. 2015;100:308-16.

15. Moore DA, Nunns M, Shaw L, et al. Interventions to improve the mental health of children and young people with long-term physical conditions: linked evidence syntheses. Health technology assessment (Winchester, England). 2019;23:1.

16. Eldridge SM, Lancaster GA, Campbell MJ, Thabane L, Hopewell S, Coleman CL, Bond CM. Defining feasibility and pilot studies in preparation for randomised controlled trials: development of a conceptual framework. PloS one. 2016 Mar 15;11(3):e0150205.

17. Bennett S, Heyman I, Coughtrey A, et al. Guided self-help interventions for mental health disorders in children with neurological conditions: study protocol for a pilot randomised controlled trial. Trials. 2016;17:532.

18. Chorpita BF, Weisz JR. Modular approach to therapy for children with anxiety, depression, trauma, or conduct problems (MATCH-ADTC). 2009.

19. Goodman R. The Strengths and Difficulties Questionnaire: a research note. Journal of child psychology and psychiatry. 1997;38:581-6. 
20. Goodman R. The extended version of the Strengths and Difficulties Questionnaire as a guide to child psychiatric caseness and consequent burden. The Journal of Child Psychology and Psychiatry and Allied Disciplines. 1999;40:791-9.

21. Attride-Stirling J. Development of methods to capture users' views of child and adolescent mental health services in clinical governance reviews (Project evaluation report). London: Commission for Health Improvement. 2003.

22. Goodman R, Ford T, Meltzer H. Mental health problems of children in the community: 18 month follow up. BMJ. 2002 Jun 22;324(7352):1496-7.

23. Goodman R, Ford T, Richards H, Gatward R, Meltzer H. The development and well-being assessment: Description and initial validation of an integrated assessment of child and adolescent psychopathology. Journal of child psychology and psychiatry. 2000;41:645-55.

24. Bennett SD, Heyman I, Coughtrey AE, et al. Assessing feasibility of routine identification tools for mental health disorder in neurology clinics. Archives of disease in childhood. 2019;104:1161-6.

25. Bennett S, Heyman I, Coughtrey A, et al. Guided self-help interventions for mental health disorders in children with neurological conditions: study protocol for a pilot randomised controlled trial. Trials. 2016;17:532.

26. Bennett SD, Coughtrey AE, Heyman I, et al. Guided self-help for mental health disorders in children and young people with chronic neurological conditions: A qualitative evaluation. European Journal of Paediatric Neurology. 2018;22(4):620-31.

27. Schulz KF, Grimes DA. Sample size slippages in randomised trials: exclusions and the lost and wayward. The Lancet. 2002 Mar 2;359(9308):781-5.

28. Nordgreen T, Haug T, Öst LG, et al. Stepped care versus direct face-to-face cognitive behavior therapy for social anxiety disorder and panic disorder: a randomized effectiveness trial. Behavior therapy. 2016 Mar 1;47(2):166-83. 
29. Catanzano M, Bennett SD, Kerry E, et al. Evaluation of a mental health drop-in centre offering brief transdiagnostic psychological assessment and treatment for children and adolescents with long-term physical conditions and their families: a single-arm, open, non-randomised trial. Evidence-Based Mental Health. 2020 Nov 26.

doi: 10.1136/ebmental-2020-300197

30. Bennett SD, Au C, Byford S, et al. Feasibility of telephone-delivered therapy for common mental health difficulties embedded in paediatric epilepsy clinics. Submitted.

31. Bennett SD, Cross HJ, Coughtrey A, et al. M.I.C.E - Mental health intervention for children with epilepsy: A randomised controlled, multi-centre clinical trial evaluating the clinical and cost-effectiveness of MATCH-ADTC in addition to usual care compared to usual care alone for children and young people with common mental health disorders and epilepsy - study protocol. Submitted. 
Table 1. Baseline demographics of GSH and WLC groups.

\begin{tabular}{|c|c|c|c|c|c|}
\hline & & & GSH $(n=17)$ & WLC $(n=17)$ & Total $(n=34)$ \\
\hline Male (\%) & & & $8(47.1)$ & $9(52.9)$ & $17(50.0)$ \\
\hline $\begin{array}{l}\text { Median Age, y (min - } \\
\text { max; IQR) }\end{array}$ & & & $\begin{array}{l}11.19(7.42- \\
18.16 ; 5.28)\end{array}$ & $\begin{array}{l}9.63(7.33- \\
16.11 ; 5.56)\end{array}$ & $11.28(3.3)$ \\
\hline \multirow[t]{7}{*}{ Additional diagnoses } & $\begin{array}{l}\text { Intellectual Disability } \\
(\%)\end{array}$ & & $7(41.2)$ & $7(41.20)$ & $14(41.2)$ \\
\hline & Autism (\%) & Diagnosis & $3(17.7)$ & $9(52.9)$ & $12(35.3)$ \\
\hline & & Symptoms & $3(17.7)$ & $2(11.8)$ & $5(14.7)$ \\
\hline & & No & $11(64.7)$ & $6(35.3)$ & $17(50.0)$ \\
\hline & $\mathrm{ADHD}(\%)$ & Diagnosis & $3(17.7)$ & $3(17.7)$ & $6(17.7)$ \\
\hline & & Symptoms & $1(5.9)$ & $0(0.00)$ & $1(2.9)$ \\
\hline & & No & $13(76.5)$ & $14(82.4)$ & $27(79.4)$ \\
\hline $\begin{array}{l}\text { Median number of } \\
\text { mental health disorder } \\
\text { diagnoses, including } \\
\text { ASD and ADHD (min- } \\
\text { max; IQR) }\end{array}$ & & & $1(1-3 ; 1)$ & $2(0-3 ; 1)$ & $2(0-3 ; 1)$ \\
\hline \multirow{2}{*}{$\begin{array}{l}\text { Primary neurological } \\
\text { diagnosis }\end{array}$} & Epilepsy (\%) & & $15(88.2)$ & $15(88.2)$ & $30(88.2)$ \\
\hline & Other $(\%)$ & & $2(11.8)$ & $2(11.8)$ & $4(11.8)$ \\
\hline \multirow{3}{*}{$\begin{array}{l}\text { Primary mental health } \\
\text { disorder }\end{array}$} & Anxiety (\%) & & $5(29.4)$ & $6(35.3)$ & $11(32.4)$ \\
\hline & Depression (\%) & & $1(5.9)$ & $2(11.8)$ & $3(8.8)$ \\
\hline & $\begin{array}{l}\text { Disruptive behaviour } \\
(\%)\end{array}$ & & $11(64.7)$ & $9(52.9)$ & $20(58.8)$ \\
\hline
\end{tabular}


Table 2. Scores on pre-post measures for GSH and WLC group at T1, T2 and follow-up for those with complete pre and post-measures

\begin{tabular}{|c|c|c|c|c|c|c|c|c|}
\hline & & & $\mathrm{T} 1$ & $\mathrm{~T} 2$ & T3 & $\begin{array}{l}\text { Within group pre- } \\
\text { post } \\
\text { Cohen's d ( } 95 \% \\
\text { CI) }\end{array}$ & $\begin{array}{l}\text { Within group } \\
\text { pre-FU } \\
\text { Cohen's d ( } 95 \% \\
\text { CI) }\end{array}$ & $\begin{array}{l}\text { Between group at } \\
\text { post } \\
\text { Cohen's d }(95 \% \\
\text { CI) }\end{array}$ \\
\hline \multirow[t]{4}{*}{ SDQ Total } & \multirow{2}{*}{$\begin{array}{l}\text { GSH } \\
(\mathrm{n}=7)\end{array}$} & Mean & 21.43 & 18.71 & 18.29 & $0.95(-0.25 ; 2.09)$ & $1.12(-0.13-2.3)$ & $0.16(-0.9 ; 1.20)$ \\
\hline & & SD & 3.259 & 2.138 & 2.138 & & & \\
\hline & \multirow{2}{*}{$\begin{array}{l}\text { WLC } \\
(\mathrm{n}=14)\end{array}$} & Mean & 22.07 & 19.29 & N/A & $0.74(-0.06 ; 1.52)$ & & \\
\hline & & SD & 3.689 & 4.697 & & & & \\
\hline
\end{tabular}




\section{Figure captions}

Figure 1. CONSORT based on completion of the primary outcome (parent completed SDQ). 